\title{
Synthesis, Characterization, and Biological Activity of 4-(2-Hydroxy-5-(aryl-diazenyl)phenyl)-6-(aryl)pyrimidin-2-ols Derivatives
}

\author{
Chandrashekhar P. Pandhurnekar, ${ }^{1}$ Ekta M. Meshram, ${ }^{2}$ \\ Himani N. Chopde, ${ }^{3}$ and Rameshkumar J. Batra ${ }^{1}$ \\ ${ }^{1}$ Department of Applied Chemistry, Shri Ramdeobaba College of Engineering and Management, Nagpur, Maharashtra 440013 , India \\ ${ }^{2}$ Department of Applied Chemistry, Guru Nanak Institute of Engineering and Technology, Kalmeshwar, Nagpur, \\ Maharashtra 440 001, India \\ ${ }^{3}$ Department of Applied Chemistry, G. H. Raisoni Academy of Engineering and Technology, Nagpur, Maharashtra 440 016, India
}

Correspondence should be addressed to Chandrashekhar P. Pandhurnekar; chand2783@gmail.com

Received 15 April 2013; Accepted 17 June 2013

Academic Editor: William Setzer

Copyright (c) 2013 Chandrashekhar P. Pandhurnekar et al. This is an open access article distributed under the Creative Commons Attribution License, which permits unrestricted use, distribution, and reproduction in any medium, provided the original work is properly cited.

\begin{abstract}
With the aim of synthesizing new heterocyclic compounds and exploring biological potency, new series of chalcones, that is, 3-(2-hydroxy-5-(aryl-diazenyl)phenyl)-1-(aryl)prop-2-en-1-one and their pyrimidine derivatives, that is, 4-(2-hydroxy-5-(aryldiazenyl)phenyl)-6-(aryl)pyrimidin-2-ols were synthesized using different aromatic amines and salicylaldehyde as starting moieties. The structures of newly synthesized compounds were confirmed using different spectroscopic techniques such as IR, ${ }^{1} \mathrm{H}-\mathrm{NMR},{ }^{13} \mathrm{C}-\mathrm{NMR}$, and mass spectral analysis, and elemental analysis. The newly synthesized pyrimidines derivatives were screened for their in vitro antibacterial and antifungal activities. It was observed that some of the newly synthesized compounds had shown promising activity against several bacterial and fungal stains. Anti-bacterial activity and anti-fungal activity studies revealed that pyrimidine derivatives consisting of nitro group in their molecular structure possess better activity than their corresponding chalcones.
\end{abstract}

\section{Introduction}

Chalcones (1,3-diaryl-2-propen-1-ones), one of the major classes of natural products belonging to the flavonoid family, have been recently the subjects of great interest for their interesting pharmacological activities $[1,2]$. In fact, the pharmacological properties of chalcones are due to the presence of both $\alpha, \beta$-unsaturation and an aromatic ring [3]. Many biological activities have been attributed to this group, such as cytotoxic $[4,5]$, antimalarial $[6,7]$, antileishmanial $[8,9]$, anti-inflammatory [10, 11], anti-HIV [12], antifungal [13], antioxidant [14], and as tyrosine kinase inhibitors [15]. Due to their abundance in plants and ease of synthesis, this class of compounds has generated great interest for possible therapeutic uses $[16,17]$. Of the many methods available for the synthesis of chalcones, the most widely used method is the base catalyzed Claisen-Schmidt reaction [18] in which the condensation of a ketone with an aldehyde is carried out in the presence of aqueous $\mathrm{NaOH}$ [19], $\mathrm{Ba}(\mathrm{OH})_{2}$ [20], $\mathrm{KOH}$, and so forth. The acid catalyzed methodologies include the use of silica sulfuric acid [21], $\mathrm{AlCl}_{3}$, dry $\mathrm{HCl}$, and so forth [22]. Chalcone derivatives are very versatile as physiologically active compounds and substrates for the evaluation of various organic syntheses. Chalcones are valuable intermediates in the synthesis of many active pharmaceutical drugs like biosynthesis of flavonoids and Auwers synthesis of flavones [23].

Pyrimidine and its derivatives are most important nitrogen based heterocycles which play a vital role in many life processes [24]. The ring system is present in nucleic acids and 
their derivatives (willardiine, tingitanine) [25] such as several vitamins (vitamin B1), antibiotics (bacimethrin, sparsomycin, bleomycin) [26], alkaloids (heteromines, crambescins, manzacidins, variolins, meridianins, psammopemmins) [27, 28], toxins [29], coenzymes, uric acid, and purines. Pyrimidine and its derivatives represents one of the important classes of heterocyclic system which is associated with the wide range of biological and pharmacological activities such as anticonvulsant [30], antimicrobial [31], anti-inflammatory [32], antiHIV [33], antitubercular [34], antitumor [35], antineoplastic [36], antimalarial [37], diuretic [38], and cardiovascular agents. Pyrimidine compounds are also used as hypnotic drugs for the nervous system [39], calcium-sensing receptor antagonists [40], and also for antagonists of the human $\mathrm{A}_{2 \mathrm{~A}}$ adenosine receptor [41].

In view of the variety of pharmacological properties exhibited by chalcones and pyrimidines, we planned to synthesize new series of chalcones, and pyrimidine derivatives. In the present communication, we thus report here the synthesis of series of chalcones that is, 3-(2-hydroxy-5-(aryl-diazenyl)phenyl)-1-(aryl)prop-2-en1-one following Claisen-Schmidt condensation reaction pathway and their pyrimidine derivatives that is 4-(2hydroxy-5-(aryl-diazenyl)phenyl)-6-(aryl)pyrimidin-2-ols (Scheme 1). Different aromatic aldehydes were synthesized as depicted earlier [42]. The structures of newly synthesized chalcones and pyrimidines compounds were confirmed using spectroscopic techniques such as ${ }^{1} \mathrm{H}-\mathrm{NMR},{ }^{13} \mathrm{C}-$ NMR, IR, and Mass spectroscopy and elemental analysis. The results of anti-bacterial and antifungal activities have also been reported here. The current investigations reveal that pyrimidine analogs exhibit better antibacterial and antifungal activities than the parent chalcone analogues.

\section{Experimental}

The chemicals and solvents were of AR grade and were used without further purification. Melting points were taken in open capillaries on TOSHNIWAL melting point apparatus and are uncorrected. IR spectra were recorded on Shimadzu Dr-8031 instrument in KBr pallets. Elemental analyses were carried out using a Perkin-Elmer, CHN elemental analyzer model 2400. ${ }^{1} \mathrm{H}-\mathrm{NMR}$ and ${ }^{13} \mathrm{C}-\mathrm{NMR}$ spectra of the synthesized compounds were recorded on a Bruker-Avance $(300 \mathrm{MHz})$ and Varian-Gemini $(200 \mathrm{MHz})$ spectrophotometer using $\mathrm{CDCl}_{3}$ solvent and TMS as an internal standard. EI-MS spectra were determined on a LCQ ion trap mass spectrometer (Thermo Fisher, San Jose, C A, USA), equipped with an EI source.

2.1. General Procedure for the synthesis of 2-Hydroxy-5((aryl)diazenyl)benzaldehyde (1a-1e). Aromatic amines $(0.01 \mathrm{moL})$ was added in conc. $\mathrm{HCl}(5 \mathrm{~mL})$ and boiled for 10 minutes. The resulting solution was then cooled to $0-5^{\circ} \mathrm{C}$ in ice bath. Aqueous sodium nitrite $\left(\mathrm{NaNO}_{2}\right)(0.01 \mathrm{moL}$, $10 \mathrm{~mL}$ ) solution in the cold condition was added in dropwise manner to this solution. The reaction mixture was then vigorously stirred. The temperature of the reaction mixture was maintained within $0-5^{\circ} \mathrm{C}$ for at least 1 hour to obtain diazonium chloride solution.

The resulting diazonium solution was then poured slowly to alkaline suspension of salicylaldehyde in water $(10 \mathrm{~mL}$, $0.01 \mathrm{moL}$ ) with continuous stirring keeping temperature within $0-5^{\circ} \mathrm{C}$. The $\mathrm{pH}$ of the reaction mixture was maintained within 8 to 10 by simultaneous addition of $10 \%$ aqueous sodium hydroxide solution. The resulting reaction mixture was kept unstirred for overnight. The obtained solid precipitate was filtered using Whatman filter paper number 40 and recrystallized using ethanol.

2.1.1. Characterization Data of 2-Hydroxy-5-(p-tolyldiazenyl)benzaldehyde (1a). Brownish Powder; Yield, 87.16\%; m.p., $115^{\circ} \mathrm{C}$; IR $(\mathrm{KBr}) \mathrm{cm}^{-1}$ : $1480(\mathrm{~N}=\mathrm{N}), 1730$ (aldehydic $\left.\mathrm{C}=\mathrm{O}\right)$, 2850 (aldehydic $\mathrm{H}-\mathrm{C}=), 2890\left(\mathrm{Ar}-\mathrm{CH}_{3}\right), 3450(\mathrm{Ar}-\mathrm{OH}) ;{ }^{1} \mathrm{H}-$ $\mathrm{NMR}\left(\mathrm{CDCl}_{3}\right) \delta: 2.35$ (s, 3H, Ar- $\left.\mathrm{CH}_{3}\right), 5.10$ (s, 1H, Ar-OH), 7.10 (s, 1H, Ar-CH), 7.30 (m, 2H, Ar-CH), 7.90 (d, 2H, Ar$\mathrm{CH}), 8.10$ (d, 1H, Ar-CH), 8.30 (s, 1H, Ar-CH), 10.30 (s, 1H, Ar-CHO); ${ }^{13} \mathrm{C}-\mathrm{NMR}\left(100 \mathrm{MHz}, \mathrm{CDCl}_{3}-\mathrm{d}_{6}, \delta, \mathrm{ppm}\right): 194.6$, $164.0,149.7,145.8,140.6,130.2,129.4,129.3,124.0,122.9,122.8$, 118.5, 116.7 (13 aromatic carbon), $21.3\left(\mathrm{CH}_{3}\right)$; Anal. Calcd. for $\mathrm{C}_{14} \mathrm{H}_{12} \mathrm{~N}_{2} \mathrm{O}_{2}$ (\%): C 69.99, H 5.03, N 11.66; Found (\%): C 69.60, H 4.90, N 11.40; MS: $m / z 240.29\left(100 \%, \mathrm{M}^{+}\right), 121.10$ (50\%), 119.00 (40\%), 93.00 (30\%), 91.10 (30\%), 29.00 (10\%), $28.00(20 \%)$.

2.1.2. Characterization Data of 2-Hydroxy-5-((2-nitrophenyl)diazenyl)benzaldehyde (1b). Brownish Powder; Yield, 78.71\%; m.p., $124^{\circ} \mathrm{C}$; IR (KBr) cm $\mathrm{cm}^{-1}$ : $1340\left(\mathrm{Ar}-\mathrm{NO}_{2}\right), 1430$ $(\mathrm{N}=\mathrm{N}$ ), 1720 (aldehydic $\mathrm{C}=\mathrm{O}$ ), 2840 (aldehydic $\mathrm{H}-\mathrm{C}=$ ), 3030 $(\mathrm{Ar}-\mathrm{OH}) ;{ }^{1} \mathrm{H}-\mathrm{NMR}\left(\mathrm{CDCl}_{3}\right) \delta: 5.20(\mathrm{~s}, 1 \mathrm{H}, \mathrm{Ar}-\mathrm{OH}), 7.30(\mathrm{~s}$, 1H, Ar-CH), 7.70-8.20 (m, 6H, Ar-CH), 10.20 (s, 1H, Ar$\mathrm{CHO}) ;{ }^{13} \mathrm{C}-\mathrm{NMR}\left(100 \mathrm{MHz}, \mathrm{CDCl}_{3}-\mathrm{d}_{6}, \delta, \mathrm{ppm}\right):$ 194.6, 164.0, $147.6,145.8,143.9,135.1,131.8,130.2,124.2$, 124.0, 123.9, 118.5, 116.7 (13 aromatic carbon); Anal. Calcd. for $\mathrm{C}_{13} \mathrm{H}_{9} \mathrm{~N}_{3} \mathrm{O}_{4}(\%)$ : C 57.57, H 3.34, N 15.49; Found (\%): C 57.40, H 3.10, N 15.20; MS: $m / z 271.18\left(100 \%, \mathrm{M}^{+}\right), 150.10(40 \%), 122.00$ (50\%), 121.20 (30\%), 93.10 (20\%), 77.10 (30\%), 45.00 (10\%), 29.20 (20\%), $28.10(10 \%)$.

2.1.3. Characterization Data of 2-Hydroxy-5-((3-nitrophenyl)diazenyl)benzaldehyde (1c). Brownish Powder; Yield, 89.59\%; m.p., $120^{\circ} \mathrm{C}$; IR (KBr) cm $\mathrm{cm}^{-1}$ : $1320\left(\mathrm{Ar}-\mathrm{NO}_{2}\right), 1420$ $(\mathrm{N}=\mathrm{N}), 1710$ (aldehydic $\mathrm{C}=\mathrm{O}$ ), 2830 (aldehydic $\mathrm{H}-\mathrm{C}=$ ), 3040 (Ar-OH); ${ }^{1} \mathrm{H}-\mathrm{NMR}\left(\mathrm{CDCl}_{3}\right) \delta: 5.40(\mathrm{~s}, 1 \mathrm{H}, \mathrm{Ar}-\mathrm{OH}), 7.50$ (s, $1 \mathrm{H}, \mathrm{Ar}-\mathrm{CH}$ ), 7.90-8.30 (m, 6H, Ar-CH), 10.40 (s, 1H, Ar$\mathrm{CHO}) ;{ }^{13} \mathrm{C}-\mathrm{NMR}\left(100 \mathrm{MHz}, \mathrm{CDCl}_{3}-\mathrm{d}_{6}, \delta, \mathrm{ppm}\right):$ 194.6, 164.0, $152.6,148.2,145.8,131.8,130.2,129.2,126.7,124.0,118.5,117.3$, 116.5 (13 aromatic carbon); Anal. Calcd. for $\mathrm{C}_{13} \mathrm{H}_{9} \mathrm{~N}_{3} \mathrm{O}_{4}(\%)$ : C 57.57, H 3.34, N 15.49; Found (\%): C 57.30, H 3.20, N 15.10; MS: $m / z 271.20\left(100 \%, \mathrm{M}^{+}\right), 151.10$ (60\%), 122.10 (50\%), 121.00 (40\%), 93.00 (10\%), 77.20 (20\%), 45.10 (10\%), 29.10 (10\%), $28.00(10 \%)$.

2.1.4. Characterization Data of 2-Hydroxy-5-((4-nitrophenyl)diazenyl)benzaldehyde (1d). Greenish Powder; Yield, 


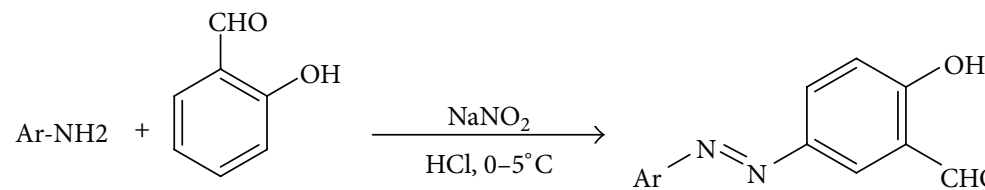

$(1 \mathbf{a}-1 \mathbf{e})$

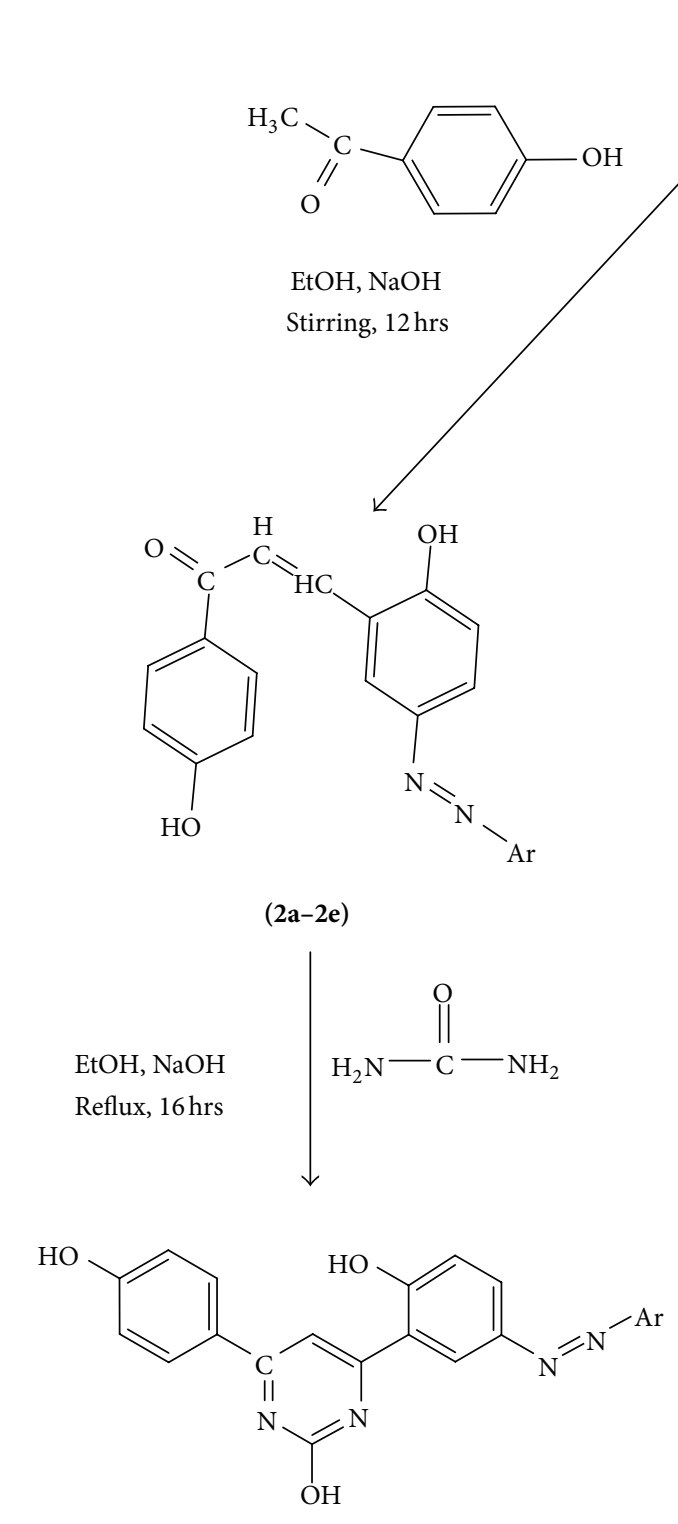

$(4 a-4 e)$

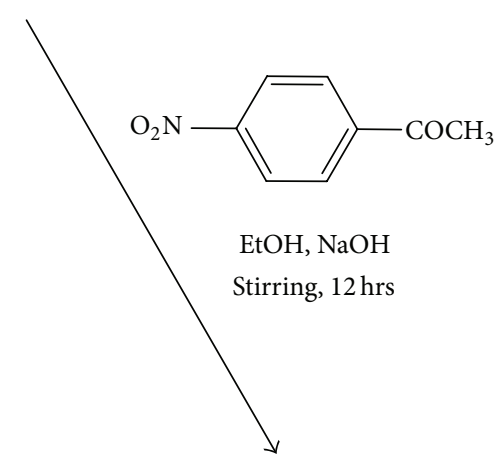<smiles>O=C(/C=C/c1cc(N=N[Al])ccc1O)c1ccc([N+](=O)[O-])cc1</smiles>

$(3 a-3 e)$

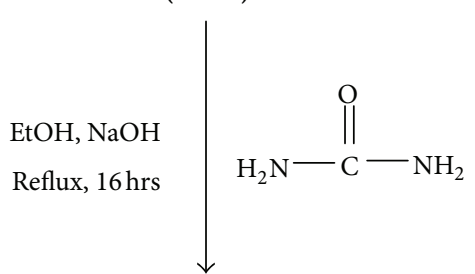

$(5 \mathbf{a}-5 \mathbf{e})$

Where, Ar- for compounds (a) $-\mathrm{C}_{6} \mathrm{H}_{4}-\mathrm{CH}_{3}$; (b) $2-\mathrm{NO}_{2}-\mathrm{C}_{6} \mathrm{H}_{4}$-; (c) $3-\mathrm{NO}_{2}-\mathrm{C}_{6} \mathrm{H}_{4}-$; (d) $4-\mathrm{NO}_{2}-\mathrm{C}_{6} \mathrm{H}_{4}-$; (e) $-\mathrm{C}_{6} \mathrm{H}_{5}$

SCHEME 1

90.32\%; m.p., $146^{\circ} \mathrm{C}$; IR (KBr) cm $\mathrm{cm}^{-1}: 1340\left(\mathrm{Ar}-\mathrm{NO}_{2}\right), 1400$ $(\mathrm{N}=\mathrm{N}), 1700$ (aldehydic $\mathrm{C}=\mathrm{O}$ ), 2345 (aldehydic $\mathrm{H}-\mathrm{C}=$ ), 2920 (Ar-OH); ${ }^{1} \mathrm{H}-\mathrm{NMR}\left(\mathrm{CDCl}_{3}\right) \delta: 5.30$ (s, 1H, Ar-OH), 7.60 (s, $1 \mathrm{H}, \mathrm{Ar}-\mathrm{CH}$ ), 7.90-8.20 (m, 6H, Ar-CH), 10.10 (s, 1H, Ar$\mathrm{CHO}) ;{ }^{13} \mathrm{C}-\mathrm{NMR}\left(100 \mathrm{MHz}, \mathrm{CDCl}_{3}-\mathrm{d}_{6}, \delta, \mathrm{ppm}\right): 194.5,164.2$, 155.8, 150.6, 145.2, 130.4, 124.3, 124.2, 124.1, 120.8, 120.7, 118.3, 116.5 (13 aromatic carbon); Anal. Calcd. for $\mathrm{C}_{13} \mathrm{H}_{9} \mathrm{~N}_{3} \mathrm{O}_{4}(\%)$ :
C 57.57, H 3.34, N 15.49; Found (\%): C 57.40, H 3.20, N 15.30; MS: $m / z 271.30\left(100 \%, \mathrm{M}^{+}\right), 150.90(70 \%), 121.80$ (40\%), 121.10 (60\%), 93.10 (30\%), 77.10 (30\%), 45.00 (20\%), $29.20(10 \%)$, $28.10(20 \%)$.

2.1.5. Characterization Data of 2-Hydroxy-5-(phenyldiazenyl)benzaldehyde (1e). Yellowish Powder; Yield, 
73.05\%; m.p., $110^{\circ} \mathrm{C}$; IR (KBr) cm $\mathrm{cm}^{-1}: 1440(\mathrm{~N}=\mathrm{N}), 1740$ (aldehydic $\mathrm{C}=\mathrm{O}$ ), 2820 (aldehydic $\mathrm{H}-\mathrm{C}=$ ), 3010 (Ar-OH); ${ }^{1} \mathrm{H}-\mathrm{NMR}\left(\mathrm{CDCl}_{3}\right) \delta: 5.20(\mathrm{~s}, 1 \mathrm{H}, \mathrm{Ar}-\mathrm{OH}), 7.10(\mathrm{~d}, 1 \mathrm{H}$, Ar- $\mathrm{CH}$ ), 7.50 (t, 3H, Ar- $\mathrm{CH}$ ), 7.90-8.10 (m, 3H, Ar-CH), 8.40 (s, 1H, Ar-CH), 10.30 (s, 1H, Ar-CHO); ${ }^{13} \mathrm{C}-\mathrm{NMR}$ $\left(100 \mathrm{MHz}, \mathrm{CDCl}_{3}-\mathrm{d}_{6}, \delta, \mathrm{ppm}\right): 194.7,164.3,152.6,145.6,130.8$, $130.5,129.6,129.5,124.1,123.2,123.1,118.7,116.5$, (13 aromatic carbon); Anal. Calcd. for $\mathrm{C}_{13} \mathrm{H}_{10} \mathrm{~N}_{2} \mathrm{O}_{2}$ (\%): C 69.02, $\mathrm{H} 4.46$, N 12.38; Found (\%): C 69.00, H 4.30, N 12.10; MS: $m / z 226.20$ (100, $\left.\mathrm{M}^{+}\right), 121.30$ (60\%), 105.10 (50\%), 93.20 (40\%), 77.20 (30\%), 29.10 (20\%), 28.20 (10\%).

2.2. General Procedure for the Synthesis of Chalcones (2a-2e, $3 a-3 e)$. To synthesize the chalcone derivatives, in the first step 2-hydroxy-5-((aryl)diazenyl)benzaldehyde (0.01 moL) and $p$-acetophenone or $p$-nitroacetophenone $(0.01 \mathrm{moL})$ were added to ethanol $(30 \mathrm{~mL})$ and then mixed thoroughly at the room temperature. In this mixture, $10 \mathrm{~mL}$ of $20 \%$ aqueous $\mathrm{NaOH}$ solution was added slowly. The reaction mixture was stirred over the magnetic stirrer for at least 12 hours. The reaction mixture was then kept for overnight. It was then poured into beaker containing crushed ice. The excess of alkali in the reaction mixture was neutralized and the reaction mixture was then slightly acidified by dropwise addition of dilute hydrochloric acid solution. The chalcone derivative, that is, 3-(2-hydroxy-5-((aryl)diazenyl)phenyl)1-(4-hydroxyphenyl)prop-2-en-1-one $(\mathbf{2 a - 2 e})$ and 3-(2hydroxy-5-((aryl) diazenyl)phenyl)-1-(4-nitrophenyl) prop-2-en-1-one $(\mathbf{3} \mathbf{a}-\mathbf{3 e})$ gets precipitated out and was filtered using Whatman filter paper number 40 . The crude chalcone product was recrystallized using ethanol.

2.2.1. Characterization Data of 3-(2-Hydroxy-5-(p-tolyldiazenyl)phenyl)-1-(4-hydroxyphenyl)prop-2-en-1-one (2a). Yellowish Powder; Yield, 77.85\%; m.p., $130^{\circ} \mathrm{C}$; IR $(\mathrm{KBr}) \mathrm{cm}^{-1}$ : $1490(\mathrm{~N}=\mathrm{N}), 1590(\mathrm{C}=\mathrm{C}), 1680(\mathrm{C}=\mathrm{O}), 2850\left(\mathrm{Ar}-\mathrm{CH}_{3}\right), 3410$ $(\mathrm{Ar}-\mathrm{OH}) ;{ }^{1} \mathrm{H}-\mathrm{NMR}\left(\mathrm{CDCl}_{3}\right) \delta: 2.30$ (s, 3H, Ar- $\left.\mathrm{CH}_{3}\right), 4.90$ (s, 2H, 2Ar-OH), 6.80-6.90 (m, 3H, Ar-CH), 7.20-7.30 (t, $3 \mathrm{H}, \mathrm{Ar}-\mathrm{CH}$ ), 7.40 (d, 2H, Ar-CH), 7.60-7.80 (m, 6H, Ar-CH); ${ }^{13} \mathrm{C}-\mathrm{NMR}\left(100 \mathrm{MHz}, \mathrm{CDCl}_{3}-\mathrm{d}_{6}, \delta, \mathrm{ppm}\right): 189.5,164.0,159.2$, $149.7,145.3,141.6,140.5,131.3,131.2,130.4,129.3,129.2,123.9$, $122.6,122.9,121.2,120.4,116.3,116.1,116.7,116.5,116.4$ (22 aromatic carbon); $21.5\left(\mathrm{CH}_{3}\right)$; Anal. Calcd. for $\mathrm{C}_{22} \mathrm{H}_{18} \mathrm{~N}_{2} \mathrm{O}_{3}$ (\%): C 73.73, H 5.06, N 7.82; Found (\%): C 73.20, H 4.90, N 7.70; MS: $m / z 358.30\left(100 \%, \mathrm{M}^{+}\right), 211.08$ (54\%), 147.05 (46\%), 121.06 (15\%), 91.10 (12\%), 93 (33\%), 28.06 (21\%), 26.10 (10\%).

2.2.2. Characterization Data of 3-(2-Hydroxy-5-((2-nitrophenyl)diazenyl)phenyl)-1-(4-hydroxyphenyl)prop-2-en-1one (2b). Brownish Powder; Yield, 67.05\%; m.p., $142^{\circ} \mathrm{C}$; IR $(\mathrm{KBr}) \mathrm{cm}^{-1}: 1320\left(\mathrm{Ar}-\mathrm{NO}_{2}\right), 1440(\mathrm{~N}=\mathrm{N}), 1580(\mathrm{C}=\mathrm{C}), 1680$ $(\mathrm{C}=\mathrm{O}), 3030$ (Ar-OH); ${ }^{1} \mathrm{H}-\mathrm{NMR}\left(\mathrm{CDCl}_{3}\right) \delta: 5.10(\mathrm{~s}, 2 \mathrm{H}$, 2Ar-OH), 6.80 (t, 3H, Ar-CH), 7.40 (d, 1H, C=CH), 7.60-7.70 $(\mathrm{m}, 7 \mathrm{H}, \mathrm{Ar}-\mathrm{CH}), 8.20(\mathrm{~d}, 1 \mathrm{H}, \mathrm{C}=\mathrm{CH}) ;{ }^{13} \mathrm{C}-\mathrm{NMR}(100 \mathrm{MHz}$, $\left.\mathrm{CDCl}_{3}-\mathrm{d}_{6}, \delta, \mathrm{ppm}\right): 189.6,164.2,159.4,147.5,145.1,143.9,141.2$, $135.2,131.6,131.2,131.1,130.4,124.4,123.8,121.2,123.5,120.4$, 116.6, 116.3, 116.1, 116.2 (21 aromatic carbon); Anal. Calcd. for $\mathrm{C}_{21} \mathrm{H}_{15} \mathrm{~N}_{3} \mathrm{O}_{5}$ (\%): C 64.73, H 3.88, N 10.79; Found (\%): C
64.70, H 3.70, N 10.60; MS: $m / z 389.38\left(100 \%, \mathrm{M}^{+}\right), 242.10$ (65\%), 147.08 (40\%), 121.05 (13\%), 122.10 (40\%), 93.10 (14\%), $28.08(21 \%), 26.10(8 \%)$.

2.2.3. Characterization Data of 3-(2-Hydroxy-5-((3-nitrophenyl)diazenyl)phenyl)-1-(4-hydroxy phenyl)prop-2-en-1-one (2c). Brownish Powder; Yield, 73.46\%; m.p., $164^{\circ} \mathrm{C}$; IR (KBr) $\mathrm{cm}^{-1}: 1310\left(\mathrm{Ar}-\mathrm{NO}_{2}\right), 1460(\mathrm{~N}=\mathrm{N}), 1570(\mathrm{C}=\mathrm{C}), 1690(\mathrm{C}=\mathrm{O})$, $3010(\mathrm{Ar}-\mathrm{OH}) ;{ }^{1} \mathrm{H}-\mathrm{NMR}\left(\mathrm{CDCl}_{3}\right) \delta: 5.20$ (s, 2H, 2Ar-OH), 6.90 (t, 3H, Ar-CH), 7.60 (d, $1 \mathrm{H}, \mathrm{C}=\mathrm{CH}), 7.80-8.10(\mathrm{~m}, 7 \mathrm{H}$, Ar-CH), 8.30 (d, $1 \mathrm{H}, \mathrm{C}=\mathrm{CH}) ;{ }^{13} \mathrm{C}-\mathrm{NMR}\left(100 \mathrm{MHz}, \mathrm{CDCl}_{3^{-}}\right.$ $\left.\mathrm{d}_{6}, \delta, \mathrm{ppm}\right): 189.6,164.6,159.1,152.3,148.5,145.5,141.3$, $131.4,131.2,131.1,130.2,129.5,126.5,123.4,121.5,120.3,117.1$, 116.7, 116.4, 116.3, 116.2 (21 aromatic carbon); Anal. Calcd. for $\mathrm{C}_{21} \mathrm{H}_{15} \mathrm{~N}_{3} \mathrm{O}_{5}$ (\%): C 64.73, H 3.88, N 10.79; Found (\%): C 64.60, H 3.60, N 10.40; MS: $m / z 389.41\left(100 \%, \mathrm{M}^{+}\right), 242.00$ (85\%), 150.10 (40\%), 147.10 (40\%), 122.10 (40\%), 121.10 (15\%), 93.10 (16\%), 77.00 (10\%), 45.10 (20\%), 28.00 (5\%), 26.00 (10\%).

2.2.4. Characterization Data of 3-(2-Hydroxy-5-((4-nitrophenyl)diazenyl)phenyl)-1-(4-hydroxy phenyl)prop-2-en-1-one (2d). Brownish Powder; Yield, 76.97\%; m.p., $188^{\circ} \mathrm{C}$; IR (KBr) $\mathrm{cm}^{-1}$ : $1330\left(\mathrm{Ar}-\mathrm{NO}_{2}\right), 1500(\mathrm{~N}=\mathrm{N}), 1580(\mathrm{C}=\mathrm{C}), 1660(\mathrm{C}=\mathrm{O})$, 3270 (Ar-OH); ${ }^{1} \mathrm{H}-\mathrm{NMR}\left(\mathrm{CDCl}_{3}\right) \delta: 5.30$ (s, 2H, 2Ar-OH), 6.60 (t, 3H, Ar-CH), 7.40 (d, 1H, C=CH), 7.70-8.10 (m, 7H, Ar-CH), 8.50 (d, $1 \mathrm{H}, \mathrm{C}=\mathrm{CH}) ;{ }^{13} \mathrm{C}-\mathrm{NMR}\left(100 \mathrm{MHz}, \mathrm{CDCl}_{3^{-}}\right.$ $\left.\mathrm{d}_{6}, \delta, \mathrm{ppm}\right): 189.4,164.4,159.2,155.4,150.3,145.3,141.4$, $131.5,131.4,130.4,124.4,124.3,123.6,120.8,120.6,120.4,116.6$, $116.5,116.4,116.3,116.1$ (21 aromatic carbon); Anal. Calcd. for $\mathrm{C}_{21} \mathrm{H}_{15} \mathrm{~N}_{3} \mathrm{O}_{5}$ (\%): C 64.73, H 3.88, N 10.79; Found (\%): C 64.40, H 3.70, N 10.60; MS: $m / z 389.10\left(100 \%, \mathrm{M}^{+}\right), 241.90$ (70\%), 151.00 (40\%), 147.00 (50\%), 121.90 (30\%), 121.00 (20\%), $92.90(20 \%), 77.10$ (12\%), 45.00 (15\%), 28.10 (10\%), 26.10 (13\%).

2.2.5. Characterization Data of 3-(2-Hydroxy-5-(phenyldiazenyl)phenyl)-1-(4-hydroxy phenyl)prop-2-en-1-one (2e). Greenish Powder; Yield, 74.06\%; m.p., $122^{\circ} \mathrm{C}$; IR (KBr) $\mathrm{cm}^{-1}$ : $1450(\mathrm{~N}=\mathrm{N}), 1550(\mathrm{C}=\mathrm{C}), 1680(\mathrm{C}=\mathrm{O}), 3020(\mathrm{Ar}-\mathrm{OH})$; ${ }^{1} \mathrm{H}-\mathrm{NMR}\left(\mathrm{CDCl}_{3}\right) \delta: 5.20(\mathrm{~s}, 2 \mathrm{H}, 2 \mathrm{Ar}-\mathrm{OH}), 6.80-7.40(\mathrm{~m}$, 10H, Ar-CH), 7.70 (s, 1H, Ar-CH), 7.90 (d, 2H, Ar-CH), 8.40 $(\mathrm{d}, 1 \mathrm{H}, \mathrm{C}=\mathrm{CH}) ;{ }^{13} \mathrm{C}-\mathrm{NMR}\left(100 \mathrm{MHz}, \mathrm{CDCl}_{3}-\mathrm{d}_{6}, \delta, \mathrm{ppm}\right)$ : $189.5,164.6,159.5,152.3,145.4,141.3,131.4,131.6,130.8,130.2$, $129.4,129.3,123.5,123.5,123.2,121.1,120.4,116.7,116.5,116.4$, 116.3 (21 aromatic carbon); Anal. Calcd. for $\mathrm{C}_{21} \mathrm{H}_{16} \mathrm{~N}_{2} \mathrm{O}_{3}$ (\%): C 73.24, H 4.68, N 8.13; Found (\%): C 73.30, H 4.50, N 8.10; MS: $m / z 344.32\left(100 \%, \mathrm{M}^{+}\right), 147.10$ (60\%), 197.08 (40\%), 121.10 (20\%), 93.90 (50\%), 77.20 (30\%), 26.30 (10\%).

2.2.6. Characterization Data of 3-(2-Hydroxy-5-(p-tolyldiazenyl)phenyl)-1-(4-nitrophenyl)prop-2-en-1-one (3a). Brownish Powder; Yield, 79.02\%; m.p., $152^{\circ} \mathrm{C}$; IR (KBr) $\mathrm{cm}^{-1}: 1350\left(\mathrm{Ar}_{-} \mathrm{NO}_{2}\right), 1510(\mathrm{~N}=\mathrm{N}), 1600(\mathrm{C}=\mathrm{C}), 1680(\mathrm{C}=\mathrm{O})$, $2850\left(\mathrm{Ar}_{-} \mathrm{CH}_{3}\right), 3380(\mathrm{Ar}-\mathrm{OH}) ;{ }^{1} \mathrm{H}-\mathrm{NMR}\left(\mathrm{CDCl}_{3}\right) \delta: 2.30$ $\left(\mathrm{s}, 3 \mathrm{H}, \mathrm{Ar}-\mathrm{CH}_{3}\right), 5.10$ (s, $\left.1 \mathrm{H}, \mathrm{Ar}-\mathrm{OH}\right), 6.80-7.20(\mathrm{~m}, 4 \mathrm{H}$, Ar-CH), 7.30 (s, 1H, C=CH), 7.40 (s, 1H, C=CH), 7.70-7.80 (t, 3H, Ar-CH), 8.10 (d, 2H, Ar-CH), 8.40 (d, 2H, Ar-CH); ${ }^{13} \mathrm{C}-\mathrm{NMR}\left(100 \mathrm{MHz}, \mathrm{CDCl}_{3}-\mathrm{d}_{6}, \delta, \mathrm{ppm}\right): 189.5,159.2,153.6$, 
$149.4,145.2,144.3,141.2,140.3,130.7,130.6,129.2,129.1,124.3$, $124.1,123.5,122.6,122.2,121.5,120.3,116.4,116.3$, (21 aromatic carbon), $21.5\left(\mathrm{CH}_{3}\right)$; Anal. Calcd. for $\mathrm{C}_{22} \mathrm{H}_{17} \mathrm{~N}_{3} \mathrm{O}_{4}$ (\%): C 68.21, H 4.42, N 10.85, Found (\%): C 68.20, H 4.30, N 10.70; MS: $m / z 387.10\left(100 \%, \mathrm{M}^{+}\right), 211.20(60 \%), 176.10(40 \%)$, 150.00 (20\%), 121.10 (40\%), 93.20 (40\%), 91.10 (30\%), 28.00 (20\%), 26.08 (10\%).

2.2.7. Characterization Data of 3-(2-Hydroxy-5-((2-nitrophenyl)diazenyl)phenyl)-1-(4-nitrophenyl)prop-2-en-1-one (3b). Brownish Powder; Yield, 73.4\%; m.p., $160^{\circ} \mathrm{C}$; IR (KBr) $\mathrm{cm}^{-1}: 1340\left(\mathrm{Ar}-\mathrm{NO}_{2}\right), 1430(\mathrm{~N}=\mathrm{N}), 1550(\mathrm{C}=\mathrm{C}), 1640(\mathrm{C}=\mathrm{O})$, 3140 (Ar-OH); ${ }^{1} \mathrm{H}-\mathrm{NMR}\left(\mathrm{CDCl}_{3}\right) \delta: 5.10$ (s, $\left.1 \mathrm{H}, \mathrm{Ar}-\mathrm{OH}\right)$, 6.90 (d, 1H, Ar-CH), 7.40 (d, 1H, C=C-H), 7.60-7.80 (m, 8H, Ar-CH), 8.10 (d, 1H, C=CH), 8.40 (d, 2H, Ar-CH); ${ }^{13} \mathrm{C}-\mathrm{NMR}$ (100 MHz, $\mathrm{CDCl}_{3}-\mathrm{d}_{6}, \delta$, ppm): 189.8, 159.6, 153.5, 147.6, 145.3, $144.1,143.7,141.3,135.4,131.5,130.6,130.5,124.6,124.3,124.2$, $123.7,123.4,121.4,120.3,116.7,116.2$ (21 aromatic carbon); Anal. Calcd. for $\mathrm{C}_{21} \mathrm{H}_{14} \mathrm{~N}_{4} \mathrm{O}_{6}$ (\%): C 60.29, $\mathrm{H}$ 3.37, N 13.39; Found (\%): C 60.20, H 3.30, N 13.10;; MS: $m / z 418.25$ (100\%, $\mathrm{M}^{+}$), 242.10 (60\%), 176.20 (50\%), 150.10 (40\%), 122.10 (30\%), 121.00 (20\%), 93.10 (10\%), 77.10 (30\%), 45.00 (10\%), 28.10 (10\%), $26.00(10 \%)$.

2.2.8. Characterization Data of 3-(2-Hydroxy-5-((3-nitrophenyl)diazenyl)phenyl)-1-(4-nitro phenyl)prop-2-en-1-one (3c). Reddish Powder; Yield, 68.01\%; m.p., $200^{\circ} \mathrm{C}$; IR ( $\left.\mathrm{KBr}\right)$ $\mathrm{cm}^{-1}$ : $1320\left(\mathrm{Ar}-\mathrm{NO}_{2}\right), 1410(\mathrm{~N}=\mathrm{N}), 1570(\mathrm{C}=\mathrm{C}), 1660(\mathrm{C}=\mathrm{O})$, 3120 (Ar-OH); ${ }^{1} \mathrm{H}-\mathrm{NMR}\left(\mathrm{CDCl}_{3}\right) \delta: 5.30$ (s, $\left.1 \mathrm{H}, \mathrm{Ar}-\mathrm{OH}\right)$, 6.60 (d, $1 \mathrm{H}, \mathrm{Ar}-\mathrm{CH}), 7.20$ (d, 1H, C=C-H), 7.40-7.60 (m, 8H, Ar-CH), 8.20 (d, $1 \mathrm{H}, \mathrm{C}=\mathrm{CH}), 8.60$ (d, $2 \mathrm{H}, \mathrm{Ar}-\mathrm{CH}) ;{ }^{13} \mathrm{C}-\mathrm{NMR}$ (100 MHz, $\mathrm{CDCl}_{3}-\mathrm{d}_{6}, \delta$, ppm): 189.5, 159.4, 153.4, 152.2, 148.4, 145.4 , 144.3, 141.7, 131.8, 130.7, 130.6, 129.3, 126.5, 124.7, 124.5, $123.7,121.8,120.4,117.1,116.5,116.4$ (21 aromatic carbon); Anal. Calcd. for $\mathrm{C}_{21} \mathrm{H}_{14} \mathrm{~N}_{4} \mathrm{O}_{6}$ (\%): C 60.29, H 3.37, N 13.39; Found (\%): C 60.10, H 3.10, N 13.20; MS: $m / z 418.30\left(100 \%, \mathrm{M}^{+}\right)$, 242.00 (70\%), 176.00 (50\%), 122.00 (40\%), 121.10 (30\%), 93.10 (10\%), 28.00 (10\%), 27.90 (10\%).

2.2.9. Characterization Data of 3-(2-Hydroxy-5-((4-nitrophenyl)diazenyl)phenyl)-1-(4-nitro phenyl)prop-2-en-1-one (3d). Greenish Powder; Yield, 70.81\%; m.p., $245^{\circ} \mathrm{C}$; IR (KBr) $\mathrm{cm}^{-1}$ : $1340\left(\mathrm{Ar}-\mathrm{NO}_{2}\right), 1500(\mathrm{~N}=\mathrm{N}), 1590(\mathrm{C}=\mathrm{C}), 1670(\mathrm{C}=\mathrm{O})$, 3250 (Ar-OH); ${ }^{1} \mathrm{H}-\mathrm{NMR}\left(\mathrm{CDCl}_{3}\right) \delta: 4.90$ (s, $\left.1 \mathrm{H}, \mathrm{Ar}-\mathrm{OH}\right)$; 6.20 (d, 1H, Ar-CH), 7.10 (d, 1H, C=C-H), 7.30-7.50 (m, 8H, Ar-CH), 8.10 (d, $1 \mathrm{H}, \mathrm{C}=\mathrm{CH}), 8.30$ (d, 2H, Ar-CH); ${ }^{13} \mathrm{C}-\mathrm{NMR}$ (100 MHz, $\left.\mathrm{CDCl}_{3}-\mathrm{d}_{6}, \delta, \mathrm{ppm}\right): 189.8,159.1,155.7,153.6,150.4$, $145.4,144.5,141.4,130.9,130.6,124.7,124.6,124.5$, 124.1, 123.7, $121.6,120.8,120.4,120.3,116.9,116.1$ (21 aromatic carbon); Anal. Calcd. for $\mathrm{C}_{21} \mathrm{H}_{14} \mathrm{~N}_{4} \mathrm{O}_{6}$ (\%): C 60.29, $\mathrm{H}$ 3.37, $\mathrm{N}$ 13.39; Found (\%): C 60.20, H 3.20, N 13.30; MS: $m / z 418.40$ (100\%, $\mathrm{M}^{+}$), 242.20 (80\%), 176.10 (70\%), 130 (50\%), 122.20 (40\%), 121.10 (30\%), 105.00 (20\%), 93.00 (10\%), 45.00 (10\%), 28.20 (10\%), 27.40 (10\%).

2.2.10. Characterization Data of 3-(2-Hydroxy-5-(phenyldiazenyl)phenyl)-1-(4-nitro phenyl)prop-2-en-1-one (3e). Brownish Powder; Yield, 80.62\%; m.p., $155^{\circ} \mathrm{C}$; IR (KBr) cm ${ }^{-1}$ :
$1310\left(\mathrm{Ar}-\mathrm{NO}_{2}\right), 1450(\mathrm{~N}=\mathrm{N}), 1580(\mathrm{C}=\mathrm{C}), 1660(\mathrm{C}=\mathrm{O}), 3150$ (Ar-OH); ${ }^{1} \mathrm{H}-\mathrm{NMR}\left(\mathrm{CDCl}_{3}\right) \delta: 5.20$ (s, 1H, Ar-OH), 6.70 (s, $1 \mathrm{H}, \operatorname{Ar}-\mathrm{CH}), 7.40$ (t, 3H, Ar-CH), 7.60 (s, 1H, CH=CH), 7.70-7.90 (m, 6H, Ar-CH), 8.10 (s, 1H, CH=CH), 8.30 (d, $2 \mathrm{H}, \mathrm{Ar}-\mathrm{CH}) ;{ }^{13} \mathrm{C}-\mathrm{NMR}\left(100 \mathrm{MHz}, \mathrm{CDCl}_{3}-\mathrm{d}_{6}, \delta, \mathrm{ppm}\right): 189.5$, $159.5,153.9,152.9,145.8,144.7,141.8,130.7,130.6,130.5,129.4$, $129.2,124.5,124.4,123.8,123.5,123.3,121.9,120.6,116.8,116.5$ (21 aromatic carbon); Anal. Calcd. for $\mathrm{C}_{21} \mathrm{H}_{15} \mathrm{~N}_{3} \mathrm{O}_{4}$ (\%): C 67.56, H 4.05, N 11.25; Found (\%): C 67.40, H 4.00, N 11.20; MS: $m / z 373.34\left(100 \%, \mathrm{M}^{+}\right), 197.10$ (60\%), 176.00 (50\%), 150 (60\%), 122.00 (50\%), 121.00 (40\%), 93.10 (20\%), 28.10 (10\%), 27.10 (10\%), 26.00 (10\%).

2.3. General Procedure for the Synthesis of Pyrimidines $(4 a-4 e, 5 a-5 e)$. A mixture of chalcone $(0.01 \mathrm{moL})$ and urea $(0.01 \mathrm{moL})$ was prepared in $30 \mathrm{~mL}$ ethanol. To this solution, $10 \mathrm{~mL} 20 \%$ aqueous $\mathrm{NaOH}$ solution was added. The resulting mixture was then refluxed on water bath for at least 16 hours. The mixture was then cooled to room temperature and poured into the beaker containing crushed ice. The solid product of pyrimidine derivatives, that is, 4-(2-hydroxy-5((aryl)diazenyl)phenyl)-6-(4-hydroxy-phenyl)pyrimidin-2ols $(4 \mathbf{a}-4 \mathbf{e})$ or 4-(2-hydroxy-5-((aryl)diazenyl)phenyl)-6-(4nitrophenyl)pyrimidin-2-ols (5a-5e) gets precipitated out. The solid obtained was filtered using Whatman filter paper no. 40 and recrystallized using ethanol.

2.3.1. Characterization Data of 4-(2-Hydroxy-5-(p-tolyldiazenyl)phenyl)-6-(4-hydroxyphenyl)-pyrimidin-2-ol (4a). Yellowish Powder; Yield, 65.18\%; m.p., $174^{\circ} \mathrm{C}$; IR ( $\left.\mathrm{KBr}\right)$ $\mathrm{cm}^{-1}$ : $1490(\mathrm{~N}=\mathrm{N}), 1610(\mathrm{C}=\mathrm{N}), 2900\left(\mathrm{Ar}-\mathrm{CH}_{3}\right), 3430 \mathrm{~cm}^{-1}$ $(\mathrm{Ar}-\mathrm{OH}) ;{ }^{1} \mathrm{H}-\mathrm{NMR}\left(\mathrm{CDCl}_{3}\right) \delta: 2.40\left(\mathrm{~s}, 3 \mathrm{H}, \mathrm{Ar}-\mathrm{CH}_{3}\right), 5.10$ (s, 3H, 3Ar-OH), 6.60 (s, 1H, Ar-CH), 6.80-7.30 (m, 7H, Ar-CH), 7.70-7.90 (m, 4H, Ar-CH); ${ }^{13} \mathrm{C}-\mathrm{NMR}(100 \mathrm{MHz}$, $\left.\mathrm{CDCl}_{3}-\mathrm{d}_{6}, \delta, \mathrm{ppm}\right): 162.7,160.4,158.5,157.6,154.9,149.5$, $145.6,140.8,129.9,129.8,128.9,128.6,128.5,124.8,123.8,122.4$, $122.6,120.9,116.8,116.7,116.3$ (21 aromatic carbon), 95.4 (C pyrimidine), $21.6\left(\mathrm{CH}_{3}\right)$; Anal. Calcd. for $\mathrm{C}_{23} \mathrm{H}_{18} \mathrm{~N}_{4} \mathrm{O}_{3}(\%)$ : C 69.34, H 4.55, N 14.06; Found (\%): C 69.30, H 4.50, N 14.00; MS: $m / z 398.42\left(100 \%, \mathrm{M}^{+}\right), 279.20(80 \%), 119.10(60 \%)$, 187.00 (50\%), 95.10 (30\%), 93.10 (20\%), 91.00 (10\%), 79.00 (10\%), 68.10 (20\%), 28.20 (10\%).

2.3.2. Characterization Data of 4-(2-Hydroxy-5-((2-nitrophenyl)diazenyl)phenyl)-6-(4-hydroxy-phenyl)pyrimidin-2-ol (4b). Brownish Powder; Yield, 54.58\%; m.p., $210^{\circ} \mathrm{C}$; IR $(\mathrm{KBr}) \mathrm{cm}^{-1}: 1310\left(\mathrm{Ar}-\mathrm{NO}_{2}\right), 1420(\mathrm{~N}=\mathrm{N}), 1670(\mathrm{C}=\mathrm{N})$, 3030 (Ar-OH); ${ }^{1} \mathrm{H}-\mathrm{NMR}\left(\mathrm{CDCl}_{3}\right) \delta: 4.90$ (s, 3H, 3Ar-OH), 6.60-6.90 (m, 4H, Ar-CH), 7.30 (d, 2H, Ar-CH), 7.70-8.40 (m, 6H, Ar-CH); ${ }^{13} \mathrm{C}-\mathrm{NMR}\left(100 \mathrm{MHz}, \mathrm{CDCl}_{3}-\mathrm{d}_{6}, \delta, \mathrm{ppm}\right)$ : $160.7,162.4,158.2,157.9,154.6,147.7,145.6,143.8,135.7131 .9$, $128.8,128.3,128.1,124.7,124.6,123.6,123.4$, 120.6, 116.6, 116.5, 116.2 (21 aromatic carbon), 95.2 (C pyrimidine); Anal. Calcd. for $\mathrm{C}_{22} \mathrm{H}_{15} \mathrm{~N}_{5} \mathrm{O}_{5}$ (\%): C 61.54, $\mathrm{H}$ 3.52, $\mathrm{N}$ 16.31; Found (\%): C 61.40, H 3.30, N 16.10; MS: $m / z 429.34\left(100 \%, \mathrm{M}^{+}\right), 307.20$ (70\%), 187.00 (50\%), 122.10 (50\%), 120.00 (40\%), 95.20 (30\%), 93.00 (20\%), $79.10(10 \%), 77.20$ (20\%), 45.10 (10\%), 68.00 (10\%), 28.10 (10\%). 
2.3.3. Characterization Data of 4-(2-Hydroxy-5-((3-nitrophenyl)diazenyl)phenyl)-6-(4-hydroxy-phenyl)pyrimidin2-ol (4c). Brownish Powder; Yield, 59.62\%; m.p., $224^{\circ} \mathrm{C}$; IR $(\mathrm{KBr}) \mathrm{cm}^{-1}$ : $1320\left(\mathrm{Ar}-\mathrm{NO}_{2}\right), 1430(\mathrm{~N}=\mathrm{N}), 1640(\mathrm{C}=\mathrm{N})$, $3010(\mathrm{Ar}-\mathrm{OH}) ;{ }^{1} \mathrm{H}-\mathrm{NMR}\left(\mathrm{CDCl}_{3}\right) \delta: 4.70(\mathrm{~s}, 3 \mathrm{H}, 3 \mathrm{Ar}-\mathrm{OH})$, 6.40-6.60 (m, 4H, Ar-CH), 7.40 (d, 2H, Ar-CH), 7.60-8.20 (m, 6H, Ar-CH); ${ }^{13} \mathrm{C}-\mathrm{NMR}\left(100 \mathrm{MHz}, \mathrm{CDCl}_{3}-\mathrm{d}_{6}, \delta\right.$, ppm): $162.8,160.9,158.3,157.1,154.4,152.9,148.1,145.7,131.2,129.4$, $128.8,128.7,128.4,126.4,124.2,123.8,120.3,117.9,116.5,116.1$, 116.3 (21 aromatic carbon), 95.9 (C pyrimidine); Anal. Calcd. for $\mathrm{C}_{22} \mathrm{H}_{15} \mathrm{~N}_{5} \mathrm{O}_{5}$ (\%): C 61.54, $\mathrm{H}$ 3.52, $\mathrm{N}$ 16.31; Found (\%): C 61.30, H 3.20, N 16.20; MS: $m / z 429.28\left(100 \%, \mathrm{M}^{+}\right), 279.10$ (60\%), 187.20 (40\%), 150.10 (30\%), 122.20 (50\%), 95.10 (30\%), 93.20 (10\%), 79.20 (20\%), 77.10 (10\%), 45.00 (10\%), 68.10 (10\%), 28.10 (10\%).

2.3.4. Characterization Data of 4-(2-Hydroxy-5-((4-nitrophenyl)diazenyl)phenyl)-6-(4-hydroxy-phenyl)pyrimidin-

2-ol (4d). Brownish Powder; Yield, 63.87\%; m.p., $240^{\circ} \mathrm{C}$; IR $(\mathrm{KBr}) \mathrm{cm}^{-1}: 1340\left(\mathrm{Ar}-\mathrm{NO}_{2}\right), 1450(\mathrm{~N}=\mathrm{N}), 1650(\mathrm{C}=\mathrm{N})$, $3400(\mathrm{Ar}-\mathrm{OH}) ;{ }^{1} \mathrm{H}-\mathrm{NMR}\left(\mathrm{CDCl}_{3}\right) \delta: 4.80(\mathrm{~s}, 3 \mathrm{H}, 3 \mathrm{Ar}-\mathrm{OH})$, 6.30-6.50 (m, 4H, Ar-CH), 7.20 (d, 2H, Ar-CH), 7.50-7.90 (m, 6H, Ar-CH); ${ }^{13} \mathrm{C}-\mathrm{NMR}\left(100 \mathrm{MHz}, \mathrm{CDCl}_{3}-\mathrm{d}_{6}, \delta, \mathrm{ppm}\right)$ : 162.2, 160.4, 158.4, 157.3, 155.4, 154.6, 150.2, 145.7, 128.9, 128.8, $128.3,124.3,124.2,124.1,123.2,120.9,120.8,120.7,116.6,116.3$, 116.2 (21 aromatic carbon), 95.1 (C pyrimidine); Anal. Calcd. for $\mathrm{C}_{22} \mathrm{H}_{15} \mathrm{~N}_{5} \mathrm{O}_{5}$ (\%): C 61.54, H 3.52, N 16.31; Found (\%): C 61.50, H 3.40, N 16.30; MS: $m / z 429.41\left(100 \%, \mathrm{M}^{+}\right), 279.20$ (70\%), 187.10 (50\%), 150.00 (40\%), 122.10 (40\%), 95.00 (20\%), 93.10 (20\%), 79.10 (10\%), 77.00 (10\%), 45.10 (10\%), 68.20 (20\%), 28.00 (10\%).

2.3.5. Characterization Data of 4-(2-Hydroxy-5-(phenyldiazenyl)phenyl)-6-(4-hydroxy-phenyl)pyrimidin-2-ol (4e). Yellowish Powder; Yield, 60.49\%; m.p., $170^{\circ} \mathrm{C}$; IR (KBr) $\mathrm{cm}^{-1}$ : $1330\left(\mathrm{Ar}-\mathrm{NO}_{2}\right), 1440(\mathrm{~N}=\mathrm{N}), 1650(\mathrm{C}=\mathrm{N}), 3070(\mathrm{Ar}-$ $\mathrm{OH}) ;{ }^{1} \mathrm{H}-\mathrm{NMR}\left(\mathrm{CDCl}_{3}\right) \delta: 4.50$ (s, 3H, 3Ar-OH), 6.60-6.80 (m, 4H, Ar-CH), 7.30 (d, 2H, Ar-CH), 7.40-7.90 (m, 7H, Ar-CH); ${ }^{13} \mathrm{C}-\mathrm{NMR}\left(100 \mathrm{MHz}, \mathrm{CDCl}_{3}-\mathrm{d}_{6}, \delta, \mathrm{ppm}\right): 162.6$, $160.1,158.9,157.7,154.8,152.3,145.4,130.8,129.7,129.4,128.6$, 128.5, 128.1, 124.7, 123.9, 123.7, 123.4, 120.1, 116.8, 116.7, 116.2, (21 aromatic carbon), 95.6 (C pyrimidine); Anal. Calcd. for $\mathrm{C}_{22} \mathrm{H}_{16} \mathrm{~N}_{4} \mathrm{O}_{3}$ (\%): $\mathrm{C}$ 68.74, $\mathrm{H}$ 4.20, N 14.58; Found (\%): C 68.60, H 4.10, N 14.30; MS: $m / z 384.32\left(100 \%, \mathrm{M}^{+}\right), 279.10$ (70\%), 187.10 (60\%), 105.10 (40\%), 95.20 (20\%), 93.10 (10\%), $79.00(10 \%), 77.20(10 \%), 45.10(10 \%), 68.10(20 \%), 28.10$ (10\%).

2.3.6. Characterization Data of 4-(2-Hydroxy-5-(p-tolyldiazenyl)phenyl)-6-(4-nitrophenyl)-pyrimidin-2-ol (5a). Brownish Powder; Yield, 70.26\%; m.p., $165^{\circ} \mathrm{C}$; $\mathrm{IR}(\mathrm{KBr}) \mathrm{cm}^{-1}$ : $1350\left(\mathrm{Ar}-\mathrm{NO}_{2}\right), 1490(\mathrm{~N}=\mathrm{N}), 1600(\mathrm{C}=\mathrm{N}), 2900\left(\mathrm{Ar}-\mathrm{CH}_{3}\right)$, $3390(\mathrm{Ar}-\mathrm{OH}) ;{ }^{1} \mathrm{H}-\mathrm{NMR}\left(\mathrm{CDCl}_{3}\right) \delta: 2.40\left(\mathrm{~s}, 3 \mathrm{H}, \mathrm{Ar}_{-} \mathrm{CH}_{3}\right)$, $5.20(\mathrm{~s}, 2 \mathrm{H}, 2 \mathrm{Ar}-\mathrm{OH}), 6.70(\mathrm{~s}, 1 \mathrm{H}, \mathrm{Ar}-\mathrm{CH}), 6.80(\mathrm{~d}, 2 \mathrm{H}$, $\mathrm{Ar}-\mathrm{CH}$ ), 7.30 (d, 2H, Ar-CH), 7.50-7.70 (m, 8H, Ar-CH); ${ }^{13} \mathrm{C}-\mathrm{NMR}\left(100 \mathrm{MHz}, \mathrm{CDCl}_{3}-\mathrm{d}_{6}, \delta, \mathrm{ppm}\right): 162.5,160.4,157.3$, 154.6, 149.6, 147.8, 145.7, 141.8, 140.5, 129.4, 129.2, 126.2, 126.1, $124.3,124.2,123.2,123.1,122.9,122.8,120.7,116.6$ (21 aromatic carbon), 95.9 (C pyrimidine), $21.2\left(\mathrm{CH}_{3}\right)$; Anal. Calcd. for $\mathrm{C}_{23} \mathrm{H}_{17} \mathrm{~N}_{5} \mathrm{O}_{4}$ (\%): C 64.63, H 4.01, N 16.39; Found (\%): C 64.40, H 4.00, N 16.20; MS: $m / z 427.10\left(100 \%, \mathrm{M}^{+}\right), 308.10$ (80\%), 187.00 (60\%), 122.10 (50\%), 119.00 (40\%), 95.10 (30\%), $93.00(10 \%), 91.10(20 \%), 79.10(10 \%), 77.10(10 \%), 45.90$ (30\%), 68.00 (10\%), 28.00 (20\%).

2.3.7. Characterization Data of 4-(2-Hydroxy-5-((2-nitrophenyl)diazenyl)phenyl)-6-(4-nitrophenyl)pyrimidin-2-ol (5b). Reddish Powder; Yield, 65.18\%; m.p., $214^{\circ} \mathrm{C}$; IR $(\mathrm{KBr}) \mathrm{cm}^{-1}$ : $1320\left(\mathrm{Ar}-\mathrm{NO}_{2}\right), 1430(\mathrm{~N}=\mathrm{N}), 1630(\mathrm{C}=\mathrm{N}), 3130(\mathrm{Ar}-\mathrm{OH})$; ${ }^{1} \mathrm{H}-\mathrm{NMR}\left(\mathrm{CDCl}_{3}\right) \delta: 5.30(\mathrm{~s}, 2 \mathrm{H}, 2 \mathrm{Ar}-\mathrm{OH}), 6.50(\mathrm{~s}, 1 \mathrm{H}$, $\mathrm{Ar}-\mathrm{CH}), 6.80$ (d, $1 \mathrm{H}, \mathrm{Ar}-\mathrm{CH}), 7.70-7.90$ (m, 10H, Ar-CH); ${ }^{13} \mathrm{C}-\mathrm{NMR}\left(100 \mathrm{MHz}, \mathrm{CDCl}_{3}-\mathrm{d}_{6}, \delta, \mathrm{ppm}\right): 162.4,160.3,157.5$, $154.3,147.9,147.5,145.7,143.7,141.5,135.3131 .7,126.5,126.4$, 124.6, 124.8, 124.7, 124.3, 123.9, 123.8, 120.6, 116.3 (21 aromatic carbon), 95.4 (C pyrimidine), Anal. Calcd. for $\mathrm{C}_{22} \mathrm{H}_{14} \mathrm{~N}_{6} \mathrm{O}_{6}$ (\%): C 57.65, H 3.08, N 18.33; Found (\%): C 57.40, H 3.00, N 18.20; MS: $m / z 458.10\left(100 \%, \mathrm{M}^{+}\right), 308.20(70 \%), 216.10$ (40\%), 222.00 (50\%), 150.00 (60\%), 122.00 (30\%), 95.00 (20\%), 93.10 (20\%), 79.00 (10\%), $68.20(20 \%)$.

2.3.8. Characterization Data of 4-(2-Hydroxy-5-((3-nitrophenyl)diazenyl)phenyl)-6-(4-nitrophenyl)pyrimidin-2-ol (5c). Brownish Powder; Yield, 54.33\%; m.p., $239^{\circ} \mathrm{C}$; IR $(\mathrm{KBr}) \mathrm{cm}^{-1}$ : $1330\left(\mathrm{Ar}-\mathrm{NO}_{2}\right), 1410(\mathrm{~N}=\mathrm{N}), 1650(\mathrm{C}=\mathrm{N}), 3030(\mathrm{Ar}-\mathrm{OH})$; ${ }^{1} \mathrm{H}-\mathrm{NMR}\left(\mathrm{CDCl}_{3}\right) \delta: 5.20(\mathrm{~s}, 2 \mathrm{H}, 2 \mathrm{Ar}-\mathrm{OH}), 6.40(\mathrm{~s}, 1 \mathrm{H}$, $\mathrm{Ar}-\mathrm{CH}), 6.90$ (d, 1H, Ar-CH), 7.60-7.90 (m, 10H, Ar-CH); ${ }^{13} \mathrm{C}-\mathrm{NMR}\left(100 \mathrm{MHz}, \mathrm{CDCl}_{3}-\mathrm{d}_{6}, \delta, \mathrm{ppm}\right): 162.2,160.6,157.3$, 154.6, 152.5, 148.8, 147.4, 145.6, 141.9, 131.9, 129.9, 126.7, 126.6, 126.1, 124.8, 124.7, 124.5, 123.4, 120.7, 117.4, 116.8 (21 aromatic carbon), 95.1 (C pyrimidine), Anal. Calcd. for $\mathrm{C}_{22} \mathrm{H}_{14} \mathrm{~N}_{6} \mathrm{O}_{6}$ (\%): C 57.65, H 3.08, N 18.33; Found (\%): C 57.50, H 2.90, N 18.10; MS: $m / z 458.38\left(100 \%, \mathrm{M}^{+}\right), 242.10$ (60\%), 216.20 (50\%), 122.10 (40\%), 121.20 (20\%), 95.10 (20\%), 93.00 (10\%), $79.10(20 \%), 68.10(10 \%)$.

2.3.9. Characterization Data of 4-(2-Hydroxy-5-((4-nitrophenyl)diazenyl)phenyl)-6-(4-nitrophenyl)pyrimidin-2-ol (5d). Brownish Powder; Yield, 61.87\%; m.p., $250^{\circ} \mathrm{C}$; IR $(\mathrm{KBr}) \mathrm{cm}^{-1}$ : $1320\left(\mathrm{Ar}-\mathrm{NO}_{2}\right), 1450(\mathrm{~N}=\mathrm{N}), 1670(\mathrm{C}=\mathrm{N}), 3420(\mathrm{Ar}-\mathrm{OH})$; ${ }^{1} \mathrm{H}-\mathrm{NMR}\left(\mathrm{CDCl}_{3}\right) \delta: 5.40(\mathrm{~s}, 2 \mathrm{H}, 2 \mathrm{Ar}-\mathrm{OH}), 6.20(\mathrm{~s}, 1 \mathrm{H}$, Ar-CH), 6.70 (d, $1 \mathrm{H}, \mathrm{Ar}-\mathrm{CH}), 7.50-7.80(\mathrm{~m}, 10 \mathrm{H}, \mathrm{Ar}-\mathrm{CH})$; ${ }^{13} \mathrm{C}$-NMR (100 MHz, $\mathrm{CDCl}_{3}-\mathrm{d}_{6}, \delta$, ppm): 162.1, 160.7, 155.7, $157.5,154.6,150.8,147.7,145.6,141.8,126.9,126.8,124.9,124.8$, $124.4,124.3,124.2,123.5,120.9,120.7,120.6,116.9$, (21 aromatic carbon), 95.7 (C pyrimidine), Anal. Calcd. for $\mathrm{C}_{22} \mathrm{H}_{14} \mathrm{~N}_{6} \mathrm{O}_{6}$ (\%): C 57.65, H 3.08, N 18.33; Found (\%): C 57.30, H 3.10, N 18.30; MS: $m / z 458.46\left(100 \%, \mathrm{M}^{+}\right), 242.20$ (80\%), 216.10 (60\%), 122.20 (50\%), 121.10 (10\%), 95.20 (10\%), 93.20 (10\%), 79.20 (10\%), 77.10 (20\%), $68.20(20 \%)$.

2.3.10. Characterization Data of 4-(2-Hydroxy-5-(phenyldiazenyl)phenyl)-6-(4-nitrophenyl)pyrimidin-2-ol (5e). Yellowish Powder; Yield, 74.22\%; m.p., $160^{\circ} \mathrm{C}$; $\mathrm{IR}(\mathrm{KBr}) \mathrm{cm}^{-1}$ : $1310\left(\mathrm{Ar}-\mathrm{NO}_{2}\right), 1420(\mathrm{~N}=\mathrm{N}), 1670(\mathrm{C}=\mathrm{N}), 3080(\mathrm{Ar}-\mathrm{OH})$; ${ }^{1} \mathrm{H}-\mathrm{NMR}\left(\mathrm{CDCl}_{3}\right) \delta: 4.90(\mathrm{~s}, 2 \mathrm{H}, 2 \mathrm{Ar}-\mathrm{OH}), 6.70(\mathrm{~s}, 1 \mathrm{H}$, Ar-CH), 7.10 (d, 1H, Ar-CH), 7.50-7.80 (m, 11H, Ar-CH); 
TABLE 1: Antibacterial and anti-fungal activity of newly synthesized chalcone derivatives (2a-2e, 3a-3e) and pyrimidine derivatives (4a-4e, 5a-5e).

\begin{tabular}{|c|c|c|c|c|c|c|}
\hline \multirow{4}{*}{ Compound ( $500 \mu \mathrm{g} /$ disk $)$} & \multicolumn{6}{|c|}{ Average value of zone of inhibition in $\mathrm{mm}$} \\
\hline & \multicolumn{4}{|c|}{ Antimicrobial Activity } & \multirow{2}{*}{\multicolumn{2}{|c|}{ Antifungal activity }} \\
\hline & \multicolumn{2}{|c|}{ Gram Positive } & \multicolumn{2}{|c|}{ Gram Negative } & & \\
\hline & S. aureus & B. subtilis & P. vulgaris & E. coli & C. albicans & A. niger \\
\hline $2 a$ & - & - & - & - & - & - \\
\hline $2 b$ & 10 & 08 & 13 & 12 & 11 & 13 \\
\hline $2 \mathrm{c}$ & 11 & 06 & 16 & 14 & 12 & 16 \\
\hline $2 d$ & 6 & 8 & 10 & 08 & 08 & 10 \\
\hline $2 e$ & - & 4 & - & - & - & - \\
\hline $3 \mathbf{a}$ & - & - & 5 & 4 & - & - \\
\hline $3 b$ & 13 & 10 & 14 & 14 & 16 & 15 \\
\hline $3 c$ & 12 & 13 & 16 & 15 & 17 & 18 \\
\hline $3 d$ & 8 & 14 & 12 & 10 & 12 & 13 \\
\hline $3 e$ & - & 5 & - & 4 & 5 & 4 \\
\hline $4 a$ & 4 & 3 & 6 & 8 & 8 & 10 \\
\hline $4 b$ & 14 & 13 & 17 & 14 & 16 & 17 \\
\hline $4 c$ & 15 & 08 & 19 & 18 & 18 & 20 \\
\hline $4 d$ & 10 & 12 & 12 & 14 & 16 & 20 \\
\hline $4 e$ & 7 & 6 & 8 & 10 & 14 & 12 \\
\hline $5 \mathbf{a}$ & 5 & 6 & 9 & 8 & 12 & 14 \\
\hline $5 b$ & 29 & 18 & 24 & 26 & 22 & 25 \\
\hline $5 c$ & 22 & 24 & 26 & 24 & 20 & 27 \\
\hline $5 d$ & 13 & 15 & 16 & 12 & 24 & 21 \\
\hline $5 e$ & 12 & 10 & 15 & 16 & 16 & 15 \\
\hline Ciprofloxacin & 30 & 26 & 32 & 31 & - & - \\
\hline Fluconazole & - & - & - & - & 24 & 26 \\
\hline
\end{tabular}

“-” represent "inactive".

${ }^{13} \mathrm{C}-\mathrm{NMR}\left(100 \mathrm{MHz}, \mathrm{CDCl}_{3}-\mathrm{d}_{6}, \delta, \mathrm{ppm}\right): 162.4,160.9,157.7$, $154.8,152.5,147.6,145.7,141.5,130.8,129.8,129.5,126.9,126.7$, $124.4,124.3,124.1,123.8,123.5,123.4,120.9$, 116.8 (21 aromatic carbon), 95.3 (C pyrimidine), Anal. Calcd. for $\mathrm{C}_{22} \mathrm{H}_{15} \mathrm{~N}_{5} \mathrm{O}_{4}$ (\%): C 63.92, H 3.66, N 16.94; Found (\%): C 63.80, H 3.50, N 16.80; MS: $m / z 413.36\left(100 \%, \mathrm{M}^{+}\right), 216.20(70 \%), 197.10$ (40\%), 122.10 (60\%), 121.00 (30\%), 95.00 (20\%), 93.10 (30\%), $77.20(20 \%), 68.10(10 \%)$.

2.4. Biological Activity. The newly synthesized compounds were examined for antibacterial and antifungal activity using well diffusion method against the panel of different gram positive and gram negative bacterial stains and fungi stains. Different bacterial stains used for the screening were $S$. aureus (gram Positive), B. subtilis (gram Positive), P. vulgaris (gram Negative), and E. coli (gram Negative). Antifungal activities of these compounds were also tested against $C$. albicans and A. niger. The stains for antibacterial and antifungal activities were obtained from Department of Microbiology, S. F. S. College, Nagpur. The stock solutions of pyrimidine derivatives or standard drug in dimethyl sulfoxide $(100 \mu \mathrm{g} / \mathrm{mL})$ were prepared for the study. The sterilized petri dishes and agar medium were used in the present work. The antibacterial activities of compounds were evaluated by measuring the zone of inhibition on nutrient agar plate. Muller Hinton agar was used in the anti-bacterial study whereas Sabouraud's Dextrose agar was used for the anti-fungal activity study. The composition of nutrient agar medium to culture the bacterial strains used in the present study was as follows: Peptone $(10 \mathrm{gm})$, agar powder $(20 \mathrm{gm})$, sodium chloride powder $(10 \mathrm{gm})$, beaf extract $(5 \mathrm{gm})$, and distilled water $(1000 \mathrm{~mL})$. The $\mathrm{pH}$ of the nutrient agar medium was adjusted to 7.2. The nutrient agar medium was mixed well and was autoclaved at $15 \mathrm{lbs}$ pressure at $120^{\circ} \mathrm{C}$ for at least 15 minutes.

In the sterilized agar medium, $10 \mathrm{~mL}$ of one-day-old bacterial/fungal cultures were added. Bacterial or fungal culture were inoculated into nutrient broth and incubated at $37 \pm 2^{\circ} \mathrm{C}$ on rotary shaker at $100 \mathrm{rpm}$. After 36-hour incubation, bacterial suspensions were used for further tests. This media were poured in petri dishes and allowed to set. Two wells were created using a $5 \mathrm{~mm}$ cork borer. In this well $0.1 \mathrm{~mL}$ of test sample/standards were filled. All the nutrient agar plates were incubated at $37^{\circ} \mathrm{C}$ for 24 hours in 
anti-bacterial study and at $37^{\circ} \mathrm{C}$ for 48 hours in anti-fungal activity study. The plates were observed for clear zone of inhibition. Then diameters of the zone of inhibition for these compounds were measured. The biological activities were tested for at least three times for all the compounds against all microorganisms and average value has been reported here. The results of antimicrobial activity and antifungal activity of the test compounds have been collected in Table 1 .

\section{Results and Discussion}

A new series of chalcones, that is, 3-(2-hydroxy-5-(aryldiazenyl)phenyl)-1-(aryl)prop-2-en-1-one (2a-2e, $\mathbf{3 a -}$ $3 \mathbf{e})$ and pyrimidines derivatives 4-(2-hydroxy-5-(aryldiazenyl)phenyl)-6-(aryl)pyrimidin-2-ols (4a-4e, 5a-5e), were synthesized as depicted in Scheme 1. In the first step, different azo-aldehydes (1a-1e) were prepared by the reaction of aromatic amines with salicylaldehyde in equimolar condition. Typical procedure of synthesis of chalcones $(\mathbf{2} \mathbf{a}-\mathbf{2} \mathbf{e}, \mathbf{3} \mathbf{a}-\mathbf{3 e})$ in the present work involves the reaction of equimolar quantities of various substituted azo-aldehydes with either 4-nitroacetophenone or p-acetophenone in ethanolic alkaline medium. Pyrimidine derivatives (4a$\mathbf{4 e}, \mathbf{5 a}-\mathbf{5 e}$ ) were obtained by refluxing newly synthesized substituted chalcones and urea in ethanol in presence of aqueous $\mathrm{NaOH}$ solution and then cooling the reaction mixture in crushed ice. The newly synthesized compounds were characterized on the basis of their spectroscopic data $\left({ }^{1} \mathrm{H}-\mathrm{NMR},{ }^{13} \mathrm{C}-\mathrm{NMR}, \mathrm{IR}\right.$, and Mass) and elemental analysis. In elemental analysis, the percentage of the nitrogen, hydrogen, and carbon was found to be experimentally equivalent to the calculated values in all compounds. In general, compounds la-1e have shown aldehydic $\mathrm{C}=\mathrm{O}$ peak within the range of $1700-1700 \mathrm{~cm}^{-1}$ whereas aldehydic $\mathrm{H}-\mathrm{C}=$ peaks approximately within 2820 up to $2850 \mathrm{~cm}^{-1}$. The ${ }^{1} \mathrm{H}-$ NMR spectra have shown the peaks of protons present in the compounds at their appropriate $\delta$ values whereas ${ }^{13} \mathrm{C}-\mathrm{NMR}$ confirmed the numbers of $\mathrm{C}$ atoms in the newly synthesized compounds. In the LCMS of the synthesized compounds, peaks for molecular ion peak were observed at its respective molecular mass. The newly synthesized chalcones and pyrimidines were evaluated for in vitro antibacterial and antifungal activities against various gram positive, gram negative bacteria, and fungal species. The results have been collected in Table 1. Ciprofloxacin as the standard drug was tested against bacterial strains whereas Fluconazole as the standard drug was tested against fungal strains for comparison with newly synthesized compounds. The close survey of values indicates that the chalcones compound $(\mathbf{2} \mathbf{a}-\mathbf{2 e}, \mathbf{3 a}-\mathbf{3 e})$ exhibited varied range zone of inhibition depending upon the substituent on the ring structure. Most of the compounds have shown moderate to good biological activity against the bacterial and fungal strains. Compounds $\mathbf{5 a}$ and $\mathbf{5 b}$ have shown excellent activity against both gram positive and gram negative bacterial strains as compared to standard drug Ciprofloxacin. Compounds $\mathbf{5 b}, \mathbf{5 c}$, and 5d have shown excellent activity against as compared to standard drug Fluconazole.

\section{Conclusion}

A series of chalcones, that is, 3-(2-hydroxy-5-(aryldiazenyl)phenyl)-1-(aryl)prop-2-en-1-one and pyrimidines derivatives 4-(2-hydroxy-5-(aryl-diazenyl)phenyl)-6-(aryl) pyrimidin-2-ols, were successfully synthesized. All the newly synthesized compounds were characterized spectroscopically and using analytical techniques such as ${ }^{1} \mathrm{H}-\mathrm{NMR},{ }^{13} \mathrm{C}-\mathrm{NMR}$, IR and mass, and elemental analysis. Anti-bacterial activity studies reveal that compounds $\mathbf{5 b}$ and $\mathbf{5} \mathbf{c}$ have shown the highest activity among all newly synthesized compounds as compared to standard drug, whereas compounds $5 \mathbf{b}, \mathbf{5 c}$, and $\mathbf{5 d}$ have shown the highest antifungal activity among all newly synthesized compounds as compared to standard drug.

\section{References}

[1] T. Narender, S. Shweta, K. Tanvir, M. Srinivasa Rao, K. Srivastava, and S. K. Puri, "Prenylated chalcones isolated from Crotalaria genus inhibits in vitro growth of the human malaria parasite Plasmodium falciparum," Bioorganic and Medicinal Chemistry Letters, vol. 15, no. 10, pp. 2453-2455, 2005.

[2] Z. Nowakowska, "A review of anti-infective and antiinflammatory chalcones," European Journal of Medicinal Chemistry, vol. 42, no. 2, pp. 125-137, 2007.

[3] M. Ceylan and E. Findik, "Synthesis and characterization of new chalcone derivatives from cis-bicyclo[3.2.0]hept-2-en-6one," Synthetic Communications, vol. 39, no. 6, pp. 1046-1054, 2009.

[4] H. O. Saxena, U. Faridi, J. K. Kumar et al., "Synthesis of chalcone derivatives on steroidal framework and their anticancer activities," Steroids, vol. 72, no. 13, pp. 892-900, 2007.

[5] R. J. Anto, K. Sukumaran, G. Kuttan, M. N. A. Rao, V. Subbaraju, and R. Kuttan, "Anticancer and antioxidant activity of synthetic chalcones and related compounds," Cancer Letters, vol. 97, no. 1, pp. 33-37, 1995.

[6] J. N. Domínguez, C. León, J. Rodrigues, N. G. De Domínguez, J. Gut, and P. J. Rosenthal, "Synthesis and evaluation of new antimalarial phenylurenyl chalcone derivatives," Journal of Medicinal Chemistry, vol. 48, no. 10, pp. 3654-3658, 2005.

[7] A. Valla, B. Valla, D. Cartier et al., "New syntheses and potential antimalarial activities of new 'retinoid-like chalcones," European Journal of Medicinal Chemistry, vol. 41, no. 1, pp. 142146, 2006.

[8] S. F. Nielsen, S. B. Christensen, G. Cruciani, A. Kharazmi, and T. Liljefors, "Antileishmaniai chalcones: statistical design, synthesis, and three-dimensional quantitative structure-activity relationship analysis," Journal of Medicinal Chemistry, vol. 41, no. 24, pp. 4819-4832, 1998.

[9] P. Boeck, C. A. Bandeira Falcão, P. C. Leal et al., "Synthesis of chalcone analogues with increased antileishmanial activity," Bioorganic and Medicinal Chemistry, vol. 14, no. 5, pp. 1538$1545,2006$.

[10] K. V. Sashidhara, M. Kumar, R. K. Modukuri et al., "Synthesis and anti-inflammatory activity of novel biscoumarin-chalcone hybrids," Bioorganic and Medicinal Chemistry Letters, vol. 21, no. 15, pp. 4480-4484, 2011. 
[11] H.-M. Yang, H.-R. Shin, S.-H. Cho et al., "Structural requirement of chalcones for the inhibitory activity of interleukin-5," Bioorganic and Medicinal Chemistry, vol. 15, no. 1, pp. 104-111, 2007.

[12] S. Cheenpracha, C. Karalai, C. Ponglimanont, S. Subhadhirasakul, and S. Tewtrakul, "Anti-HIV-1 protease activity of compounds from Boesenbergia pandurata," Bioorganic and Medicinal Chemistry, vol. 14, no. 6, pp. 1710-1714, 2006.

[13] L. Svetaz, A. Tapia, S. N. López et al., "Antifungal chalcones and new caffeic acids esters from Zuccagnia punctata acting against soybean infecting fungi," Journal of Agricultural and Food Chemistry, vol. 52, no. 11, pp. 3297-3300, 2004.

[14] B. P. Bandgar, B. S. Hote, N. A. Dhole, and R. N. Gacche, "Synthesis and biological evaluation of novel series of chalcone derivatives as inhibitors of cyclooxygenase and LPS-induced TNF- $\alpha$ with potent antioxidant properties," Medicinal Chemistry Research, vol. 21, pp. 2292-2299, 2011.

[15] O. Nerya, R. Musa, S. Khatib, S. Tamir, and J. Vaya, "Chalcones as potent tyrosinase inhibitors: the effect of hydroxyl positions and numbers," Phytochemistry, vol. 65, no. 10, pp. 1389-1395, 2004.

[16] K. V. Sashidhara, A. Kumar, M. Kumar, J. Sarkar, and S. Sinha, "Synthesis and in vitro evaluation of novel coumarinchalcone hybrids as potential anticancer agents," Bioorganic and Medicinal Chemistry Letters, vol. 20, no. 24, pp. 7205-7211, 2010.

[17] C. T. Hsieh, T. J. Hsieh, M. El-Shazly et al., "Synthesis of chalcone derivatives as potential anti-diabetic agents," Bioorganic \& Medicinal Chemistry Letters, vol. 22, pp. 3912-3915, 2012.

[18] S. Wattanasin and W. S. Murphy, "An improved procedure for the preparation of chalcones and related enones," Synthesis, vol. 1980, pp. 647-650, 1980.

[19] T. Narender, K. Venkateswarlu, B. V. Nayak, and S. Sarkar, "A new chemical access for $3^{\prime}$-acetyl- $4^{\prime}$-hydroxychalcones using borontrifluoride-etherate via a regioselective Claisen-Schmidt condensation and its application in the synthesis of chalcone hybrids," Tetrahedron Letters, vol. 52, no. 44, pp. 5794-5798, 2011.

[20] A. Garcia-Raso, J. A. Garcia-Raso, R. Mestres, and J. V. Sinisterra, "Mechanism of Michael addition of ethyl acetoacetate to chalcone catalyzed by activated $\mathrm{Ba}(\mathrm{OH})_{2}$," Reaction Kinetics and Catalysis Letters, vol. 28, no. 2, pp. 365-371, 1985.

[21] J. R. Patel, M. H. Malani, and B. Z. Dholakiya, "Silica sulfuric acid-catalyzed Claisen-Schmidt condensation of 1,3,4 trisubstituted pyrrole 2,5 dione to chalcones," Research on Chemical Intermediates, vol. 38, pp. 2371-2381, 2012.

[22] K. V. Sashidhara, J. N. Rosaiah, and A. Kumar, "Iodine-catalyzed mild and efficient method for the synthesis of chalcones," Synthetic Communications, vol. 39, no. 13, pp. 2288-2296, 2009.

[23] A. K. Verma and R. Pratap, "Chemistry of biologically important flavones," Tetrahedron, vol. 68, pp. 8523-8538, 2012.

[24] M. H. Bukhari, H. L. Siddiqui, M. Ahmad, T. Hussain, and M. G. Moloney, "Synthesis and anti-bacterial activities of some novel pyrazolobenzothiazine-based chalcones and their pyrimidine derivatives," Medicinal Chemistry Research, vol. 21, pp. 28852895, 2011.

[25] E. A. Bell and R. G. Foster, "Structure of lathyrine," Nature, vol. 194, no. 4823, pp. 91-92, 1962.
[26] F. Tanaka, S. Takeuchi, N. Tanaka, H. Yonehara, H. Umezawa, and Y. J. Sumiki, "Bacimethrin, a new antibiotic produced by B. megatherium," Journal of Antibiotics A, vol. 14, pp. 161-162, 1961.

[27] R. G. S. Berlinck, J. C. Braekman, D. Daloze et al., "Polycyclic guanidine alkaloids from the marine sponge Crambe crambe and $\mathrm{Ca}++$ channel blocker activity of crambescidin 816," Journal of Natural Products, vol. 56, no. 7, pp. 1007-1015, 1993.

[28] Y.-L. Lin, R.-L. Huang, C.-M. Chang, and Y.-H. Kuo, "Two new puriniums and three new pyrimidines from Heterostemma brownii," Journal of Natural Products, vol. 60, no. 10, pp. 982985, 1997.

[29] I. Ohtani, R. E. Moore, and M. T. C. Runnegar, "Cylindrospermopsin: a potent hepatotoxin from the blue-green alga Cylindrospermopsis raciborskii," Journal of the American Chemical Society, vol. 114, pp. 7941-7942, 1992.

[30] N. Jiang, X. Q. Deng, F. N. Li, and Z. S. Quan, "Synthesis of novel 7-substituted-5-phenyl-[1, 2, 4] triazolo[1, 5-a] pyrimidines with anticonvulsant activity," Iranian Journal of Pharmaceutical Research, vol. 11, pp. 799-806, 2012.

[31] M. Ungureanu, C. C. Moldoveanu, A. Poeata, G. Drochioiu, M. Petrovanu, and I. Mangalagiu, "New pyrimidine compounds with in vitro antimicrobial or antifungal activity," Annales Pharmaceutiques Françaises, vol. 56, pp. 181-183, 1998.

[32] A. E.-G. E. Amr, N. M. Sabry, and M. M. Abdulla, "Synthesis, reactions, and anti-inflammatory activity of heterocyclic systems fused to a thiophene moiety using citrazinic acid as synthon," Monatshefte fur Chemie, vol. 138, no. 7, pp. 699-707, 2007.

[33] N. Fujiwara, T. Nakajima, Y. Ueda, H. Fujita, and H. Kawakami, "Novel piperidinylpyrimidine derivatives as inhibitors of HIV1 LTR activation," Bioorganic and Medicinal Chemistry, vol. 16, no. 22, pp. 9804-9816, 2008.

[34] L. Ballell, R. A. Field, G. A. C. Chung, and R. J. Young, "New thiopyrazolo[3,4-d]pyrimidine derivatives as antimycobacterial agents," Bioorganic and Medicinal Chemistry Letters, vol. 17, no. 6, pp. 1736-1740, 2007.

[35] E. Wagner, K. Al-Kadasi, M. Zimecki, and W. SawkaDobrowolska, "Synthesis and pharmacological screening of derivatives of isoxazolo[4,5-d]pyrimidine," European Journal of Medicinal Chemistry, vol. 43, no. 11, pp. 2498-2504, 2008.

[36] A. Tiwari, W. R. Waud, and R. F. Struck, "Determination of the phamacophore of penclomedine, a clinically-evaluated antitumor pyridine derivative," Bioorganic and Medicinal Chemistry, vol. 10, no. 11, pp. 3593-3598, 2002.

[37] K. Görlitzer, S. Herbig, and R. D. Walter, "Indeno[1,2d]pyrimidin-4-yl-amines," Pharmazie, vol. 52, no. 9, pp. 670672,1997

[38] I. V. Ukrainets, I. A. Tugaibei, N. L. Bereznyakova, V. N. Kravchenko, and A. V. Turov, "4-Hydroxy-2-quinolones. 144. Alkyl-, arylalkyl-, and arylamides of 2-hydroxy-4-oxo-4Hpyrido[1,2-a]pyrimidine-3-carboxylic acid and their diuretic properties," Chemistry of Heterocyclic Compounds, no. 5, pp. 718-729, 2008

[39] S. Q. Wang, L. Fang, X. J. Liu, and K. Zhao, "Design, synthesis, and hypnotic activity of pyrazolo[1,5-a]pyrimidine derivatives," Chinese Chemical Letters, vol. 15, no. 8, pp. 885-888, 2004.

[40] W. Yang, Z. Ruan, Y. Wang et al., "Discovery and structureactivity relationships of trisubstituted pyrimidines/pyridines as 
novel calcium-sensing receptor antagonists," Journal of Medicinal Chemistry, vol. 52, no. 4, pp. 1204-1208, 2009.

[41] R. J. Gillespie, S. J. Bamford, R. Botting et al., "Antagonists of the human A2A adenosine receptor. 4. Design, synthesis, and preclinical evaluation of 7-aryltriazolo[4,5-d]pyrimidines," Journal of Medicinal Chemistry, vol. 52, no. 1, pp. 33-47, 2009.

[42] H. A. Deshpande, H. N. Chopde, C. P. Pandhurnekar, and R. J. Batra, "Synthesis, characterization and testing of biological activity of some novel chalcones derivatives of coumarin," Chemical Science Transactions, vol. 2, pp. 621-627, 2013. 

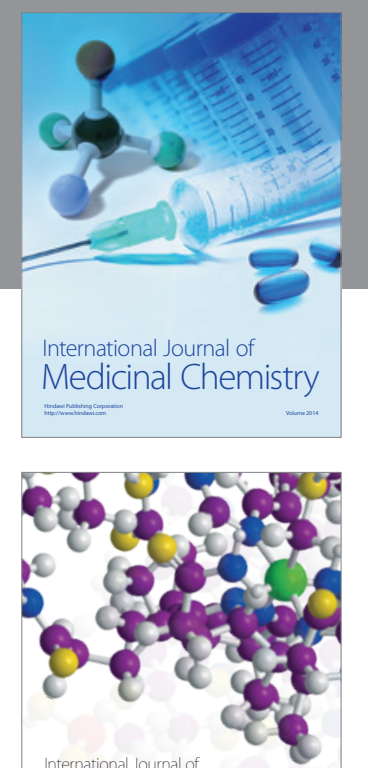

\section{Carbohydrate} Chemistry

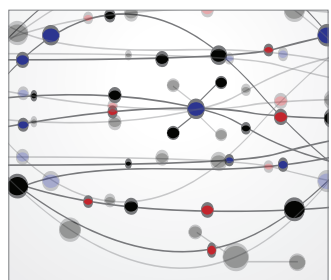

The Scientific World Journal
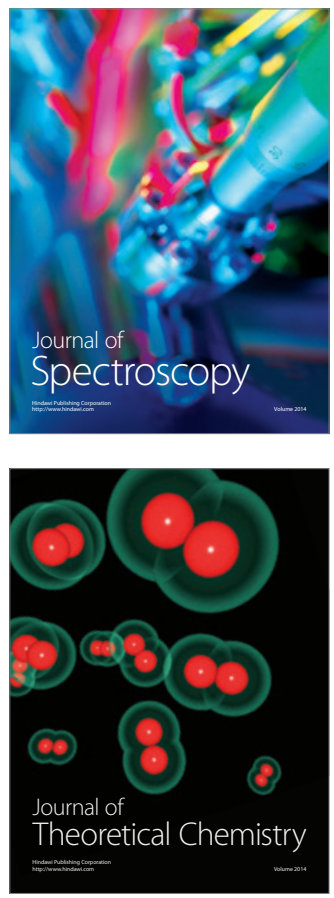
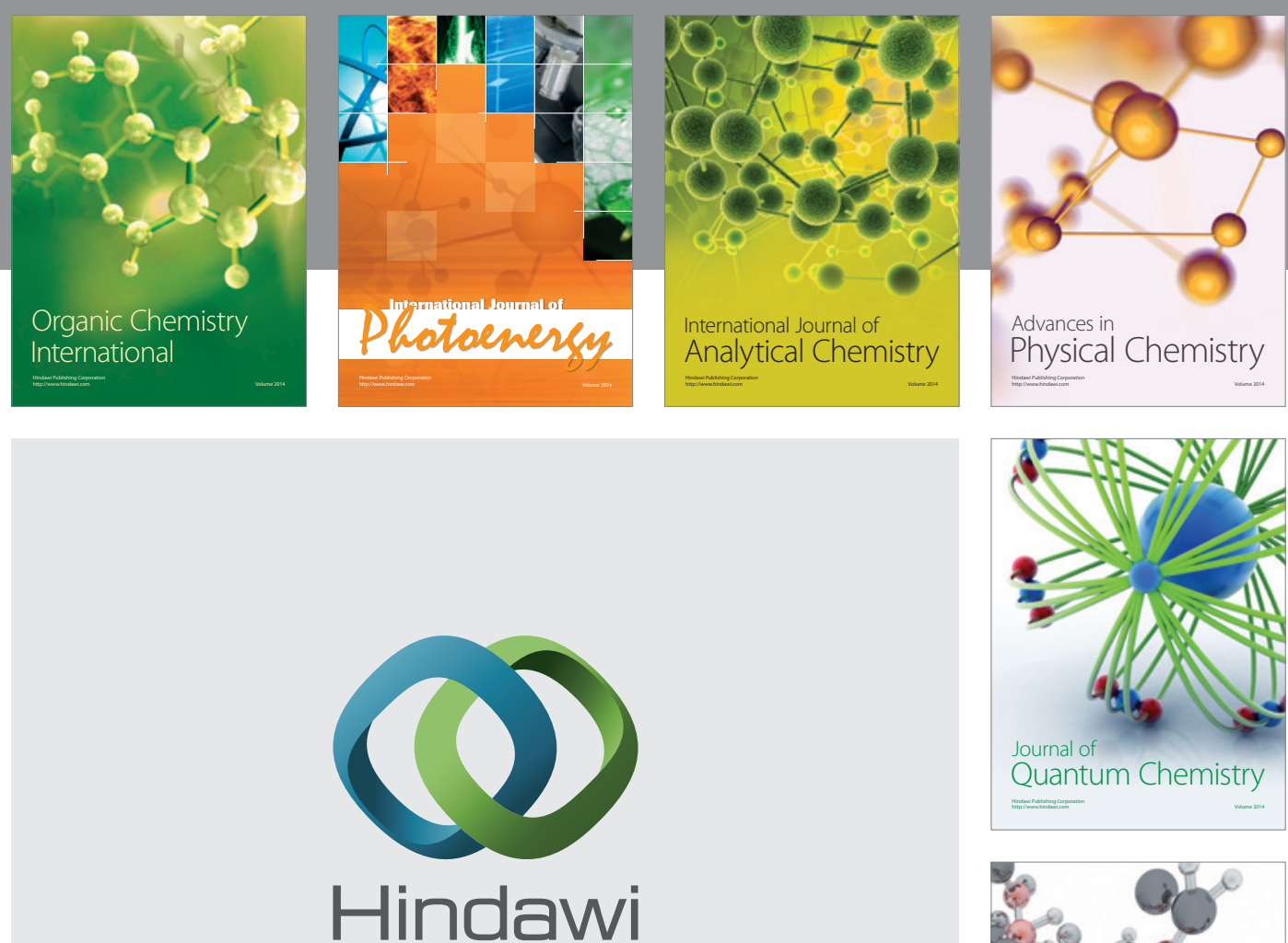

Submit your manuscripts at

http://www.hindawi.com

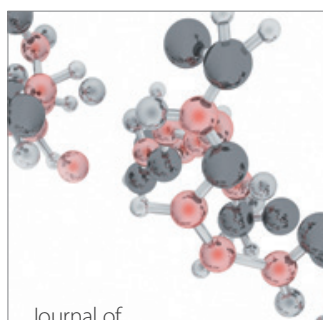

Analytical Methods

in Chemistry

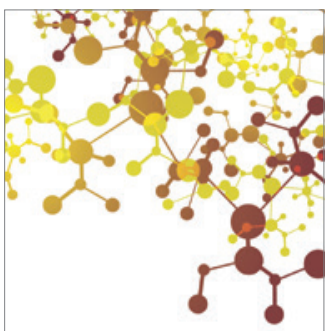

Journal of

Applied Chemistry

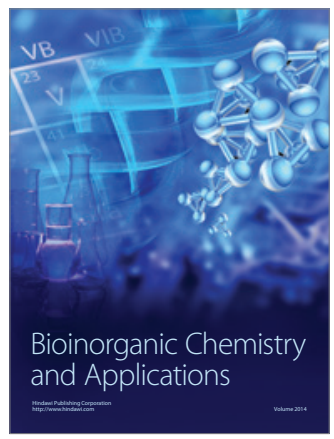

Inorganic Chemistry
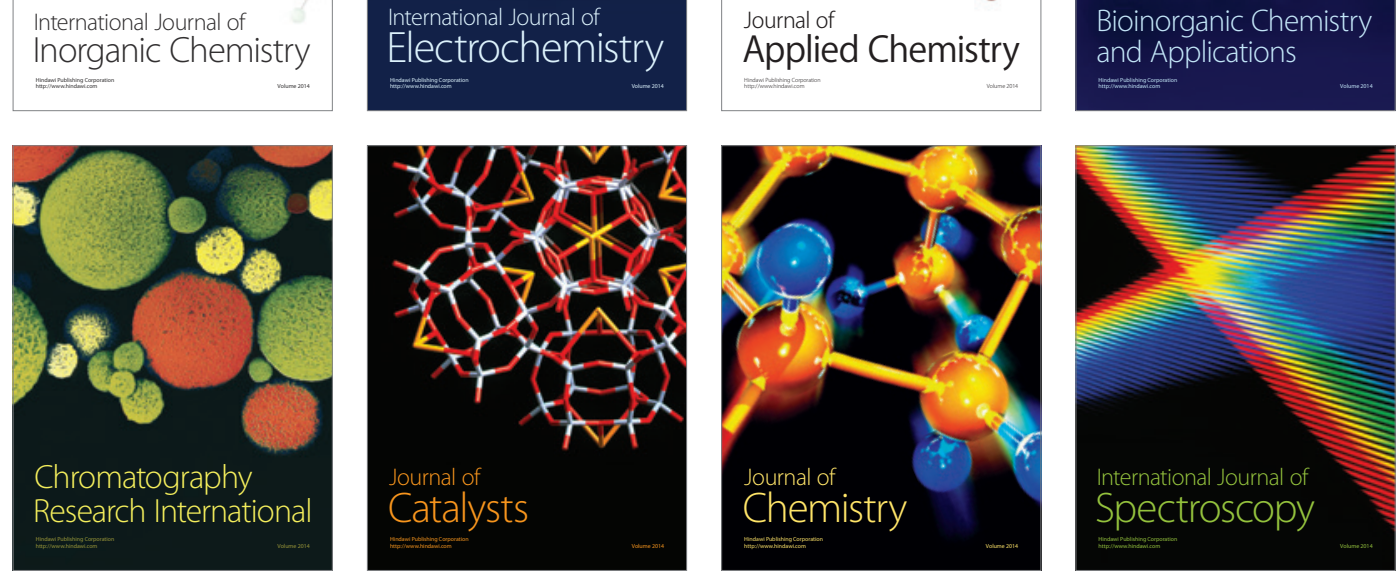